\title{
La teneur du lait de vache en nitrate
}

\author{
par \\ B. REMOND \\ Laboratoire de la production laitière \\ C.R.Z.V. de Theix - 63110 Beaumont
}

Un nombre relativement important d'études portant sur le nitrate chez les plantes et chez les animaux a été réalisé (cf. revues de Wright et Davison, 1964, 205 références, et de Becker, 1967, 165 références). Chez les premières, elles ont surtout porté sur les facteurs d'accumulation, tandis que chez les seconds, elles se sont principalement attachées aux effets toxiques consécutifs à l'ingestion de nitrate.

Très peu d'études ou de mesures ont cependant concerné la teneur du lait en nitrate. Même quand ils ont expérimenté avec des vaches en lactation, les auteurs ne s'y sont pas toujours intéressés (cf. Morris et al., 1958 ; Stewart et Merilan, 1958 ; Jones et al., 1966 ; Farra et Satter, 1971 ; Murdock et Hodgson, 1972). Cependant compte tenu d'une part de la place importante occupée par le lait de vache dans l'alimentation humaine, et d'autre part des effets toxiques observés chez les bovins à la suite de fortes ingestions de nitrate, il nous a semblé intéressant de compiler les données disponibles afin d'évaluer la possibilité, dans certaines conditions, que le lait contienne des quantités substantielles de nitrate, susceptibles de nuire à la santé du consommateur.

\section{TENEUR NORMALE DU LAIT EN NITRATE}

Des quelques travaux réalisés depuis la fin du siècle dernier jusqu'à une époque assez récente, il ne ssemblait pas possible à Davis et Mc Donald (1953) de dégager la preuve définitive de la présence de nitrate dans le lait. Il semble que la disparité des résultats était due au manque de spécificité et de sensibilité des méthodes de mesures utilisées.

Dans cet article les concentrations de nitrate sont exprimées en concentrations de $\mathrm{NO}_{3}$. 
Avec une méthode qu'il avait lui-même mise au point et qui permet de mesurer des concentrations de $0,2 \mathrm{ppm}$, Hänni (1954) a mesuré pendant 1 an la teneur en nitrate de deux échantillons de lait mensuels prélevés dans le tank d'une laiterie industrielle. Il a enregistré une teneur moyenne en nitrate de $0,66 \mathrm{ppm}$ et une teneur maximale de 1,7 ppm. Avec la méthode à la diphénylamine, aucune trace n'était décelable. Signalons aussi, au passage, que d'après des essais effectués par ce même auteur, ni la pollution du lait par les bouses, ni les maladies de la mamelle n'augmentent la teneur du lait en nitrate.

Avec un régime constitué de foin et d'aliment concentré, pauvres en nitrate, distribué à des génisses, Davison et al. (1964) ont enregistré une teneur en nitrate du lait de $5 \mathrm{ppm}$.

Enfin, sur les 200 premiers échantillons de lait (150 échantillons de lait d'étable et 50 échantillons de lait de laiterie) prélevés sur l'ensemble de la France dans le cadre d'une enquête menée par l'Institut Technique de l'Elevage Bovin, la teneur moyenne en nitrate se situe aux environs de 0,3 ppm, et la teneur maximale enregistrée est de 1,1 ppm (résultats partiels, Mahieu, communication personnelle).

\section{INFLUENCE DE L'INGESTION DE QUANTITES ELEVEES DE NITRATE}

L'influence de l'ingestion de quantités élevées de nitrate sur la teneur en nitrate du lait a donné lieu à quelques expérimentations dont les résultats sont rassemblés dans le tableau ci-après. Voici les principales conditions dans lesquelles ils ont été obtenus :

Davison et al. (1964) ont pulvérisé une solution de nitrate de sodium sur le foin distribué à des génisses, de façon à leur faire ingérer $440 \mathrm{mg}$ ou $660 \mathrm{mg}$ de nitrate par $\mathrm{kg}$ de poids vif et par jour. Un lot d'animaux recevait le foin tel quel.

Hänni (1954) a distribué à 4 vaches les différents régimes suivants :

$1^{\circ} 14 \mathrm{~kg}$ de foin $+2 \mathrm{~kg}$ d'aliment concentré $+14 \mathrm{~kg}$ de betteraves (teneur des betteraves en nitrate : $490 \mathrm{ppm}$ ).

$2^{\circ}$ Même régime que précédemment, mais augmentation de la quantité de betteraves jusqu'à $22 \mathrm{~kg}$.

$3^{\circ}$ Foin + aliment concentré $+35 \mathrm{~kg}$ de navettes (teneur des navettes en nitrate : $450 \mathrm{ppm}$ ).

4. Herbe jeune, jusqu'à $80 \mathrm{~kg}$ par jour (teneur de l'herbe en nitrate : $180 \mathrm{ppm})$.

Le lait était collecté dans des récipients nettoyés avec de l'eau dépourvue de nitrate. 
Influence de la quantité de nitrate ingérée sur la teneur du lait en nitrate

\begin{tabular}{|c|c|c|}
\hline Auteurs & $\begin{array}{c}\mathrm{NO}_{3} \text { ingéré } \\
(\mathrm{mg} / \mathrm{kg} \text { de poids vif/jour) }\end{array}$ & $\begin{array}{c}\mathrm{NO}_{3} \text { dans le lait } \\
(\mathrm{ppm})\end{array}$ \\
\hline Davison et al. (1964) (1) & $\begin{array}{r}0 \\
440 \\
660\end{array}$ & $\begin{array}{r}5 \\
9 \\
15\end{array}$ \\
\hline Hänni (1954) (2) & 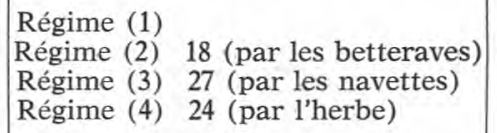 & $\begin{array}{c}\text { Non décelé } \\
》 \\
》 \\
》\end{array}$ \\
\hline Sebaugh et al. (1970) (3) & $\begin{array}{r}37 \text { (110 p. } 100 \text { énergie) } \\
370 \text { ( } 80 \text { p. } 100 \text { énergie }) \\
(110 \text { p. } 100 \text { énergie })\end{array}$ & $\begin{array}{l}4,33 \\
3,50 \\
2,50\end{array}$ \\
\hline $\begin{array}{l}\text { Nebraska Health Officials } \\
\text { (1950) cité par Wright et } \\
\text { Davison (1964) }\end{array}$ & $505 \mathrm{ppm} \mathrm{NO}_{3}$ dans eau de boisson & Non décelé \\
\hline
\end{tabular}

(1) Aux quantités indiquées de nitrate ingérées (qui correspondent au nitrate pulvérisé), il faudrait ajouter environ $9 \mathrm{mg}$ (par $\mathrm{kg}$ de poids vif et par jour) apportés par les aliments. La production laitière des vaches était d'environ $19 \mathrm{~kg}$.

(2) Les quantités de nitrate ingérées ont été calculées en estimant un poids vif de $600 \mathrm{~kg}$.

(3) La production laitière des vaches était d'environ $11 \mathrm{~kg}$. L'ion nitrite n'a pu être décelé dans le lait.

Sebaugh et al. (1970) ont étudié avec 6 vaches, dans un essai en carré latin $3 \times 3$ de courte durée (10 semaines au total), l'influence de la quantité de nitrate ingérée (37 ou $370 \mathrm{mg}$ par $\mathrm{kg}$ de poids vif) et du niveau énergétique de la ration ( 80 ou 110 p. 100 des recommandations de Morrison).

Enfin, les services officiels de santé du Nébraska ont mesuré la teneur en nitrate du lait produit par des vaches dont l'eau de boisson avait une teneur en nitrate de 505 ppm.

Dans 3 des 4 essais ou enquêtes, l'ingestion de quantités importantes de nitrate n'a pas eu d'influence sur la teneur du lait en cet ion (Nebraska Health Officials, 1950 ; Hänni, 1954 ; Sebaugh et al., 1970). Néanmoins, dans l'essai de Davison et al. (1964), la teneur du lait en nitrate a augmenté avec la quantité de nitrate ingérée par les animaux. Il faut cependant signaler que dans cet essai le nitrate était apporté de façon artificielle (par pulvérisation sur le fourrage) et que les apports étaient particulièrement élevés puisque dans le lot qui en recevait le plus, 2 vaches sont mortes et 1 a failli mourir. 
Il faut enfin peut-être faire une réserve concernant ces résultats. Selon Murdock et Hodgson (1972), les tentatives généralement infructueuses de reproduction des symptômes de toxicité dus au nitrate suggèrent que des facteurs physiologiques inconnus contribuent à leur apparition. S'il en était bien ainsi, cela pourrait conférer un caractère artificiel aux essais réalisés et en diminuer (mais dans quelle mesure ?) la valeur.

\section{DISCUSSION ET CONCLUSION}

Le faible nombre d'expérimentations et de mesures portant sur la teneur du lait en nitrate laisse à penser qu'elle n'a jamais constitué un problème sanitaire sérieux, mais il rend toute conclusion définitive risquée. Il apparaît néanmoins que la teneur en nitrate du lait non contaminé par apport de nitrate après la traite est très faible (de l'ordre de grandeur du ppm), et que la teneur parfois plus élevée du lait échantillonné au niveau des industries est due au nitrate contenu dans l'eau de lavage des récipients, du moins dans les régions où la teneur de l'eau en nitrate est notable. Même quand la santé des vaches a été atteinte à la suite d'une ingestion massive de nitrate (Davison et al., 1964), la teneur du lait en cet ion (15 ppm) a été bien inférieure à la teneur limite prescrite pour l'eau potable : 44 ppm (Code de la Santé Publique, Arrêté du 10 août 1961).

Les faibles teneurs du lait en nitrate s'expliquent assez bien par la physiologie de la vache laitière :

$1^{\circ}$ Au niveau du rumen, la majeure partie du nitrate ingéré est transformée par les micro-organismes, en nitrite puis en ammoniac (Lewis, 1951). L'accumulation de nitrite dans le rumen proviendrait de ce que, au-dessus d'une certaine teneur du jus de rumen en nitrite, la transformation de ce dernier en ammoniac serait plus lente que celle du nitrate en nitrite. Il en résulte que, contrairement au monogastrique, le ruminant n'absorbe que partiellement le nitrate qu'il ingère, une partie du reste passant dans le sang sous forme d'ammoniac et de nitrite (Li Chuan Wang, 1961), ce qui provoque d'ailleurs les effets toxiques parfois observés. Dans l'essai de Davison et al. (1964) il est remarquable que les vaches présentaient des symptômes d'empoisonnement bien avant que la teneur du lait en nitrate n'atteigne la valeur limite admise pour l'eau destinée à la consommation humaine.

$2^{\circ} \mathrm{Au}$ niveau de la glande mammaire, le nitrate ne semble pas filtrer librement du plasma sanguin au lait, comme c'est le cas pour l'urée (Journet, Vérité, Vignon, 1975), puisque sa teneur est de 3,5 à 10,5 fois plus élevée dans le plasma sanguin que dans le lait (Sebaugh et al., 1970) : la glande mammaire semble jouer le rôle d'une barrière. 
Remarque concernant la teneur du lait en nitrite: En ce qui concerne la teneur du lait en nitrite, le nombre de références disponible est encore plus faible que pour celle en nitrate. Selon Davis et Mc Donald (1953) du lait auquel du nitrate a été ajouté présente généralement une réaction au nitrite après 2 jours. Dans l'essai réalisé par Sebaugh et al. (1970), le lait ne contenait pas de nitrite, qu'elle qu'ait été la quantité de nitrate ingérée. Il faut cependant remarquer que la teneur en nitrite du plasma sanguin était égale à zéro, et que la proportion de méthémoglobine était inférieure à 5 p. 100. Enfin, dans l'enquête actuellement menée par l'Institut Technique de l'Elevage Bovin, 2 échantillons sur les 200 qui ont été analysés jusqu'à présent avaient une teneur en nitrite de $0,1 \mathrm{ppm}$, les autres n'en contenant pas, ou seulement des traces non quantifiables (Mahieu, communication personnelle).

\section{S u m m a r y}

The author reviews the data concerning the level of nitrate in cow's milk. This data is scarce and their values depend largely on the author cited. This data shows nevertheless that the level of nitrate in milk is very low (in the range of p.p.m. of $\mathrm{NO}_{3}$ ). When the cows ingest a large quantity of nitrate, the level in milk of this ion may increase, but it still stays below the level admissible by the Official Recommendations for drinking water, even when the cows show signs of nitrite poisoning. The level of this ion in milk is either not measurable or is very low (approx. 0,1 p.p.m.). The level in milk of nitrate is discussed in the light of our knowledge of nitrate metabolism in ruminants.

Reçu pour publication en mars 1975.

\section{Références bibliographiques}

Becker (M.) (1967). - Nitrat und Nitrit in der Tierernährung (ein Ubersichtsreferat). Qualitas. Pl. Mater, veg., 15, 48-64.

Davis (J. G.), Mc Donald (F. J.) (1953). - Richmond's Dairy Chemistry. 5e édition, p. 408, Charles Griffin Company Limited, Londres.

Davison (K. L.), Hansel (W. M.), Krook (L.), Mc Entee (K.), Wright (M. J.) (1964), - Nitrate toxicity in dairy heifers. I. Effects on reproduction, growth, lactation and vitamin A nutrition. J. Dairy Sci., 47, 1065-1073.

FARRA (P. A.), SATTER (L. D.) (1971). - Manipulation of Ruminal fermentation. III. Effect of nitrate on Ruminal volatile fatty acid production and milk composition. J. Dairy Sci., 54, 1018-1024.

HanNi (H.) (1954), - Uber den Nitratnachweis in Milch. Mitt. Lebensm. Hyg., Bern., 45, (6), 502-508. 
Jones (I. R.), Weswig (P. H.), Bone (J. F.), Peters (M. A.), Alpan (S. O.) (1966). Effect of high nitrate consumption on lactation and vitamine A Nutrition of dairy cows. J. Dairy Sci., 49, 491-499.

Journal Officiel de la République Française. Lois et Décrets, $\mathrm{n}^{\circ}$ 200, Samedi 26 août 1961, p. 8033.

Journet (M.), VéRité (R.), Vignon (B.) (1975). - L'azote non protéique du lait. Facteurs de variation. Le Lait, 543-544, p. 212.

Lewis (D.) (1951). - The metabolism of nitrate and nitrite in the sheep. 1. The reduction of nitrate in the rumen of the sheep. Biochem. J., 48, 175-180.

Li Chuan Wang, Garcia-Rivera (J.), Burris (R. H.) (1961). - Metabolism of nitrate by cattle. Biochem. J., 81, 237-242.

Mahieu. - Institut Technique de l'Elevage Bovin. Communication personnelle.

Morris (M. P.), Cancel (B.), Gonzalez-Mas (A.) (1958). - Toxicity of nitrates and nitrites to dairy cattle. J. Dairy Sci., 41, 694-696.

Murdock (F. R.), Hodgson (A. S.) (1972). - Utilization of nitrates by dairy cows. J. Dairy Sci., 55, 640-642.

Sebaugh (T. P.), Lane (A. G.), Campbell (J. R.) (1970). - Effects of two levels of nitrate and energy on lactating cows receiving urea. J. Anim. Sci., 31, 142-144.

Stewart (G. A.), Merilan (C.P.) (1958). - Effect of potassium nitrate intake on lactating dairy cows. Missouri Univ. Agr. Expt. Sta. Res. Bull., 650, 1-11.

Wright (M. J.), Davison (K. L.) (1964). - Nitrate accumulation in crops and nitrate poisoning in animals. Adv. Agron., 16, 197-247. 